\title{
Unidad en la diferencia: Reflexiones sobre el derecho cosmopolita *
}

\author{
THOMAS McCARTHY \\ Northwestern University, Evanston
}

El artículo intenta abordar una evaluación del modelo normativo de relaciones internacionales bosquejado recientemente por John Rawls. Para ello subraya sus virtudes (la sensibilidad al acomodamiento, reconocimiento y respeto de la diversidad cultural) y sus defectos (el achatamiento de su horizonte normativo) mediante el contraste con la propuesta de otro teórico político contemporá- neo que también bebe de las fuentes kantianas del ideal cosmopolita: Jürgen Habermas. El autor concluye con una propuesta en la que la diversidad cultural y los ideales normativos cosmopolitas son integrados más allá de la completa aceptación de la facticidad y de la total abstracción universalista insensible al contexto.

Immanuel Kant, al comentar en 1795 el sistema de Estados inaugurado por la Paz de Westfalia ciento cincuenta años antes, expresaba recelos que nos son familiares respecto al principio de soberanía estatal y al derecho de los Estados a recurrir a las armas para proteger sus intereses vitales, dos rasgos que han seguido siendo pilares del Derecho internacional del siglo xx: «considerado el derecho de gentes como un derecho a la guerra, resulta en realidad inconcebible; porque habría de concebirse entonces como un derecho a determinar lo justo y lo injusto, no según leyes exteriores de valor universal limitativas de la libertad de cada individuo, sino según máximas parciales, asentadas sobre la fuerza bruta. Sólo hay un modo de entender ese derecho a la guerra, y es el siguiente: que es muy justo y legítimo que quienes piensan de ese modo se destrocen unos a otros y vayan a buscar la paz perpetua en el seno de la tierra, en la tumba, que con su manto fúnebre tapa y cubre los horrores y los causantes de la violencian" ${ }^{1}$. $\mathrm{Y}$ a continuación propone la idea del pacifismo legal, que aún hoy es la principal alternativa al estado de naturaleza en las relaciones internacionales: «Para los Estados, en sus mutuas relaciones, no hay, en razón, ninguna otra manera de salir de la situación anárquica, origen de continuas guerras, que sacrificar, como hacen los individuos, su salvaje libertad sin freno y reducirse a públicas leyes coactivas, constituyendo así un Estado de naciones - civitas gentium - que, aumentando sin cesar, legue por fin a contener en su seno todos los pueblos de la tierra» ${ }^{2}$. Pero de inmediato concede la imposibilidad de un Estado mundial y propone en su lugar una liga

* Traducciốn de Ángel Rivero.

'Immanuel Kant, «La paz perpetua», en Lo bello y lo sublime. La paz perpetua, traducción de A. Sinchez Rivero y F. Rivera Pastor, Colección Austral, Madrid, Espasa-Calpe, 1982, p. 112.

Ibid., pp. 112-113. 
permanente de naciones, una idea que ha modelado los acuerdos internacionales posteriores a la guerras mundiales de este siglo: «Pero si no quieren esto, por la idea que tienen del derecho de gentes (...) entonces, para no perderlo todo, en lugar de la idea positiva de una república universal puede acudirse al recurso negativo de una fedcración de pueblos que, mantenida y extendida sin cesar, evite las guerras y ponga un freno a las tendencias perversas e injustas, aunque siempre con el peligro constante de un estallido irreparable» ${ }^{3}$. Esta última cláusula podría servir para recordarnos, si aún necesitamos que nos lo recuerden, las debilitadoras limitaciones que han sufrido tales acuerdos y su consiguiente fracaso a la hora de controlar de forma efectiva el recurso a la violencia, y la amenaza de la violencia, en el conflicto internacional. Como mucho - por ejemplo, en la Carta de las Naciones Unidas y la Declaración Universal de Derechos Humanos- se ha comenzado a relativizar el principio de soberanía estatal en el derecho internacional, pero no se ha reemplazado - como queda evidentemente demostrado por la composición del Consejo de Seguridad de la ONU, con cinco miembros permanentes con derecho de veto-. Las consecuencias de esto para la paz mundial, por no hablar de la justicia mundial, sólo se ha evidenciado de forma clara en las últímas décadas.

Así, en lo atinente al Derecho internacional estamos aún en una situación que es, en muchos aspectos, y desde el punto de vista teórico, muy parecida a la de Kant, En muchos aspectos, pero desde luego no en todos. La formulación clásica de Kant de la idea liberal de derecho se centraba únicamente en la igual libertad individual bajo leyes generales. Al escribir antes del surgimiento de las democracias de masas - después de todo, el sufragio universal para los adultos es una conquista del siglo $\mathrm{xx}$ - $\mathrm{y}$ antes de que fueran notorias las consecuencias clasistas del desarrollo capitalista, podía aceptar la monarquía constitucional como la mejor forma de gobierno republicano y quedar fiado y tranquilo de que una sociedad de propietarios privados, dejada a su aire, haría avanzar la justicia social. Hoy, una teoría normativa del Derecho internacional habría de combinar su preocupación liberal por la libertad individual bajo la ley con una preocupación democrática por la participación, por la representación y por la responsabilidad y con una preocupación igualitaria por la justicia social. No voy a intentar demostrar aquí, puesto que no es mi tema, que esas preocupaciones están íntimamente relacionadas — de hecho, hablando conceptualmente, lo están «internamente»-. Permítanme, simplemente, que haga observar que la interdependencia teórica es parte integral de las dos teorías de la justicia neo-kantianas más poderosas de que ahora disponemos, las de John Rawls y Jürgen Habermas ${ }^{4}$. Por supuesto, esto sólo hace más apremiante la preocupación de Kant acerca de la realizabilidad del ideal de un derecho cosmopolita. Si la salvaguarda efectiva de los derechos humanos básicos a travếs del imperio internacional de la ley está aún lejos de haberse alcanzado, las formas transnacionales de autodeterminación democrática y redistribución igualitaria parecen aún más lejanas. Pero las oportunidades y los obstáculos para la

${ }^{3}$ ibid, p. 113.

- Véanse John Rawls, A Theory of Justice, Harvard U. P., Cambridge, 1971, y Political Liberalism, Nueva York, Columbia U. P., 1993, y Jürgen Habermas, Between Facts and Noms, The Mit Press, 1996. 
realización de tal ideal tampoco son mi tema, aunque mis observaciones estarán modeladas por una preocupación general por la practicabilidad de las concepciones normativas de la justicia internacional, más allá de la cual devendrían meramente "utópicas» en el sentido negativo del término. El eje de mis observaciones está, por tatto, en otro sitio que ahora paso a explicar.

Hoy día, los problemas filosóficos más peliagudos para los ideales cosmopolitas del siglo xviI, como el de Kant, tienen su origen en los desafíos abiertos, en casi todos los frentes, al racionalismo, universalismo y progresismo ilustrados que los animaban. Bajo el peso del colonialismo de los siglos $\mathrm{xIX}$ y $\mathrm{xx}$, muchos han considerado tales ideales como meros apoyos culturales del imperialismo occidental. Y el germen neocolonial incubado en los procesos de descolonización posteriores a la Segunda Guerra Mundial ha tendido a desacreditar, como ideologias neoimperialistas, las teorías de la modernización en las que habían sido reconstruidos. Ha devenido inequívocamente claro que las formas históricamente dominantes de tales ideas e ideales, putativamente trascendentes, estaban en realidad construidas socialmente y estaban profundamente relacionadas con el poder y el interés. La cosa no recibió mucha ayuda en sus reformulaciones marxistas, porque quedaron igualmente desacreditadas por el ascenso y caída del socialismo realmente existente durante este siglo. En los últimos años la principal respuesta a este estado de cosas ha sido un asalto total al universalismo eurocéntrico, y junto a esto, la celebración de las diferencias culturales. En este espíritu, la política de la identidad y de la diferencia adopta típicamente la forma de una oposición de lo particular a lo univeral, de la multiplicidad real de culturas a la presunta unicidad de la razón, a menudo con el argumento de que los principios y las prácticas de la razón no pueden trascender las fronteras culturales. Una de las implicaciones de esto, para la política internacional, parece clara: la autonomía local ha de defenderse frente a los modelos metropolitanos de un «nuevo orden mundial».

Al mismo tiempo, sin embargo, no es difícil discernir huellas de ideas cosmopolitas en las críticas postcoloniales a la globalización neocolonial. Estas críticas descansan a menudo, más o menos, en el mismo tipo de impulso universalista expresado por las ideas recibidas de igual respeto, igual dignidad, igual tratamiento, autodeterminación, etc. ${ }^{5}$. Y es que las acusaciones de eurocentrismo sólo pueden concitar la fuerza moral que se les supone viniendo de tal fuente. Es más, los teóricos postcoloniales rechazan hoy, de forma creciente, el «organicismo» de las concepciones de la cultura que se encuentra tras muchas de las críticas al universalismo, como se ha visto con claridad por lo fácil que es que tales concepciones se pongan al servicio de elites nacionalizadoras. Las ideologías del retorno a las auténticas tradiciones, costumbres e identidades han sido utilizadas, como hemos visto, con demasiada frecuencia para suprimir a otros indígenas - disidentes, mujeres, minorías étnicas, etc.- como para mantener su inocencia intelectual. Por tanto, los teóricos de la «hibridización» cultural son con frecuencia tan críticos del organicismo multicultural como lo son del universalismo monocultural ${ }^{6}$. Consideran

\footnotetext{
${ }^{5}$ Desarrollo este argumento en el contexto de la antropología kpostmoderna en *Doing the Right Thing in Cross-Cultural Representation», Ethics, 102 (1992), pp. 635-649.

- Véase, por ejemplo, James Clifford, The Predicament of Culture, Harvard U. P., Cambridge, 1988, y Homi Bhabha, The Location of Culture, Nueva York, Routledge, 1994.
} 
que la cultura global no es ni una concatenación de mónadas culturales ni es un proceso de convergencia cultural.

El colonialismo y el neocolonialismo han impulsado, indudablemente, un proceso de homogeneización cultural. $\mathrm{Y}$, ciertamente, las tendencias globalizadoras recientes lo han intensificado. ¿Podría ser de otra manera con más de un billón de televisores en funcionamiento, con facilidades de acceso cada vez mayores, vía satélite, a las ofertas de los gigantes de la comunicación y, por tanto, a imágenes mediadas por la comunicación de masas de su identidad, de su sociedad y de sus proyectos de vida personal y social; con la rápida globalización de todo tipo de emisiones y material impreso; con el continuo tejer global de internet y el constante flujo de correo electrónico más allá de las fronteras nacionales; con migraciones masivas de gentes; con la creación de públicos desterritorializados, transnacionales; con la urbanización explosiva de poblaciones hasta ahora rurales; con la globalización de la producción económica y el consumo, que hace que la gente de todo el planeta sueñe con las mismas fantasías de abundancia material? Pero también se ha vuelto evidente que mientras los flujos culturales erosionan algunas formas de heterogeneidad, también producen nuevos híbridos, y que aunque hay constantes préstamos, éstos no se producen en una sola dirección y rara vez dan lugar a fotocopias. En suma, no hay un peligro inminente de que desaparezcan las diferencias; pero tampoco permanecerán estadísticamente iguales, en una especie de meseta inmóvil de modelos tradicionales. Los repertorios culturales locales cambian a medida que las masas, y no sólo las elites, van ganando experiencia de la diversidad de formas modernas?.

Estoy recordando estos rasgos generales de nuestra situación con la única intención de sugerir que los tiempos estân maduros para repensar los ideales cosmopolitas más allá de la oposición abstracta entre unidad y diferencia, de forma más particular, para reemplazar el universalismo monocultural de las versiones clásicas con un universalismo multicultural más sensible a la dialéctica de lo general y lo particular. Por supuesto, estoy lejos de ser el primero en haber sugerido esto. Tanto en los estudios postcoloniales como en la teoría política abundan tales sugerencias ${ }^{8}$. Desafortunadamente, los primeros han tendido a quedarse exclusivamente en el plano cultural y, por tanto, no se han ocupado adecuadamente de tematizar los requisitos institucionales y procedimentales de la justicia cosmopolita. Y los últimos han tendido a poner tan en primer plano los temas legales y políticos que las cuestiones culturales han quedado abandonadas en un trasfondo sin problematizar. Pero esto

"Véase Arjun Appadurai, Modernity at Large. Cultural Dimensions of Globalization, Filadelfia, L. of Pennsylvania Press, 1996. La revista Public Culture, publicada por la University of Chicago Press para la Society for Transnational Culture Studies, publica con regularidad artículos sobre este tema y temas conexos.

* Pheng Cheah desarrolla una consistente defensa de la necesidad de repensar el cosmopolitismo en los estudios postcoloniales en kGiven Culture: Rethinking Cosmopolitan Freedom in Transnationalism», manuscrito no publicado, aunque sus razones para hacerlo apuntan $\mathrm{cn}$ una direccion algo distinta de la que aquí se ha tomado. Janna Thompson argumenta, de forma convincente, la necesidad de rcpensar el cosmopolitismo en la teoria política en Justice and the World Order, Londres, Routledge, 1992, aunque de nuevo la línea que toma no es la que tomamos aquí. Thompson ofrece un buen repaso de las teorias tradicionales y contemporáneas de la justicia internacional. 
está cambiando. La diferencia cultural se ha convertido recientemente en una preocupación principal de la corriente dominante de la teoría política. De hecho, es una preocupación central de los dos tcóricos que antes mencioné. Habcrmas, en Between Facts and Noms, y Rawls, en Political Liberalism, son absolutamente conscientes, de hecho dolorosamente conscientes, de las dificultades que lo irreducible de la diversidad presenta para sus reformulaciones de la concepción kantiana de la justicia. Estos dos trabajos están dedicados, principalmente, al constitucionalismo en una sociedad singular, pero multicultural, y ambos autores han ofrecido también propuestas sobre cómo extender sus concepciones de la justicia nacional a concepciones de la justicia global ${ }^{9}$. Las diferencias entre las dos propuestas son muy instructivas, porque señalan dos caminos distintos, que divergen en puntos importantes, para ir del universalismo monocultural al universalismo multicultural en la teoría legal y política $1 "$.

Los problemas de lo uno y lo múltiple son tan viejos como la filosofía misma. En la era moderna, los esfuerzos por pensar la unidad en la diferencia se centraban en la idea de razón, y en el ámbito de la filosofía política en la idea del argumento razonado, por ejemplo en la forma del contrato social. Para Kant, era función de la razón generar principos de unidad sistemática, en el caso de la razón práctica, la unidad de los agentes racionales bajo las leyes comunes que éstos se daban a sí mismos. Su idea de una asociación de seres libres e iguales bajo el imperio de leyes que todos pudieran razonablemente aceptar sigue siendo fundamental para los proyectos de Rawls y Habermas. Las diferencias interesantes aparecen en las formas en las que ambos tratan de acomodar lo que Rawls llama kel hecho del pluralismo razonable», un hecho que la noción dieciochesca de Kant de la cultura racional le permitía ignorar en su tiempo. En términos generales, Habermas permanece todo lo próximo posible al proyecto kantiano como permite el reconocimiento de este hecho, que él interpreta, sobre todo, en términos ético-culturales, esto es, de nuevo hablando en general, en términos de lo que Hegel denominó Sittlichkeit. Su estrategia es construir una dialéctica de lo universal - a saber, la unidad de la razón práctica- y de lo particular —es decir, la diversidad de la vida ética- con el fin de llegar a lo que podríamos denominar un «universalismo sensible al contexton, capaz de acomodar un haz indeterminado de particularidades limitadas. Tendré ocasión de decir algo más sobre este enfoque en la última sección de este artículo.

Rawls, por contra, adopta la estrategia, hablando en general, de buscar un territorio político-legal común en medio de la diversidad ideológica y cultural. De forma sorprendente, propone un giro copernicano en el pensamiento del contrato social al incluir entre los apoyos de la tolerancia las diferencias mismas de concepciones filosóficas de la razón, de la verdad, del significado y de valores,

\footnotetext{
Véase John Rawk, "The Law of Peoples», en S. Shute y S. Hurley, eds, On Human Rights. The Oxford Amnesty Lecturss 1993, Nueva York, Basic Books, 1993, pp. 41-82, y Jürgen Habermas, Die Einbeziehung des Anderen, Suhrkamp, Francfort, 1996.

${ }^{16}$ Las diferencias brsicas ya eran evidentes en sus enfoques acerca de la justicia en el ámbito nacional, como intenté mustrar en kKantian Constructivism and Reconstructivism: Rawls and Habermas in Dialoguew, Ethics, 105 (1994), pp. 44-63.
} 
que subyacen a las concepciones rivales de la justicia política en nuestra tradición. Es una propuesta valiente y descansa sobre algunas consideraciones harto poderosas. Si una estructura común es un sine qua non del imperio de la ley, y si la legitimidad democrática requiere del consentimiento informado y razonado de los gobernados, y si la gente razonable es tan diversa en términos culturales, ideológicos y filosóficos, entonces no puede haber imperio legítimo de la ley a menos que reconozcamos las «cargas de la razón» y busquemos el acuerdo político en este lado, al margen de todos los desacucrdos razonables y persistentes accrea de cuestiones no políticas. La irreductible pluralidad de visiones básicas acerca del significado y valor de la vida humana hace necesario construir una concepción puramente «política» de la justicia que «quede libre» de las «doctrinas generales» de todo tipo. Esto está desde luego lejos del llamado de Kant en favor de una crítica ilustrada que someta todas las demandas a la autoridad del libre examen de la razón "i; y de hecho es posible localizar las principales diferencias entre Rawls y Habermas a lo largo del espectro de opciones que van desde la tolerancia al acomodamiento y de la crítiça a la transformación. Primero consideraré la estrategia de Rawls de keludir» la construcción de la unidad política en la diferencia cultural.

«Las personas razonables», nos dice Rawls en tono kantiano, «desean para sí un mundo social en que, como libres e iguales, puedan cooperar con los otros en términos que todos puedan aceptar» $(\mathrm{PL} 50)^{12}$. Pero el otro aspecto que define lo «razonable», tal como to entiende, es una «diposición a reconocer la carga de la prueba y a aceptar sus consecuencias para el uso de la razón pública en la dirección del ejercicio legítimo del poder público en un régimen constitucional» (PL 54). Reconocer la carga de la prueba significa entender por qué los desacuerdos razonables entre personas razonables no son sólo posibles sino probables, esto es, entender por qué son un resultado normal del libre uso de la razón, incluso a largo plazo y en circunstancias favorables. A la luz de las muchas fuentes y causas de tales desacuerdos es irracional esperar que «las personas conscientes,

" Nuestra época es, de modo especial, la de la crítica. Todo ha de someterse a ella. Pero la religión y la legislación pretenden de ordinario escapar a la misma. La primera, a causa de la santidad y la segunda, a causa de su majestad. Sin embargo, al hacerlo despiertan contra sí mismas sospechas justificadas y no pueden exigir un respeto sincero, respeto que la razón sólo concede a lo que es capaz de rosistir un examen público y libre», Immanuel Kant, Crítica de la Razón Pura, Madrid, Alfaguara, 1978, trad. de Pedro Ribas, «Prólogo a la primera edición, A XI, nota de Kant. En la introducción a Political Liberalism Rawls desautoriza explícitamente el "proyecto ilustrados de encontrar una base racional general para el liberalismo (PL xviii). En «Two Concepts of Liberalism» [Ethics 105 (1995), pp. 516-534], Willian Galston argumenta que Political Liberalism deja a Rawls más cerca del «Proyecto Ilustrado"; predicado sobre la autonomia, y más lejos del "Proyecto de la Reforma», predicado sobre la diversidad, de lo que Rawls quisiera. A este respecto Galston se hace eco de las dudas acerca de la presunta neutralidad del liberalismo politico planteadas por Samuel Seheffler y Leif Wenar en las ensayos que se citan en la nota 20 .

${ }^{17}$ Political Liberalism será citado en paréntesis en el texto como PL. 
plenas en sus capacidades mentales, tras libre discusión, arriben [siempre] a las mismas conclusiones» (PL 58). Estilizándolo algo, podríamos contemplar estos dos aspectos de lo razonable como si estuvieran en una tensión más o menos análoga a las dos perspectivas que Kant distingue en la Fundamentación y en otros sitios. Como agentes racionales, deseamos justificar nuestras acciones ante otros mediante fundamentos que todos puedan aceptar racionalmente. Como espectadores u observadores, sin cmbargo, sc nos hace cvidente el hecho del pluralismo razonable y anticipamos que algunas de las razones aceptables para nosotros pueden ser inaceptables para otros. ¿Cómo se pueden combinar estas dos posiciones accrca de la justificación pública? La estrategia de Rawls es descontar por adelantado el pluralismo, por decirlo de alguna manera, al restringir la «concepción política de la justicia» al ámbito de un «consenso solapado»: «la justicia como equidad se orienta a desvelar una base pública de justificación acerca de cuestiones de justicia política dado el hecho del pluralismo razonable. Puesto que la justificación se dirige a otros, procede desde lo que es o puede ser tenido en común, y así comenzamos con las ideas fundamentales compartidas implicitas en la cultura política pública con la esperanza de desarrollar desde ellas una concepción política que pueda obtener un acuerdo libre y razonado en el juicio, este acuerdo será estable en virtud de que gane el apoyo de un consenso solapado de doctrinas generales razonables» (PL 63). La «intención práctica» de obtener una base pública de justificación (PL 9) motiva la estrategia de comenzar con ideas implícitamente compartidas y convertirlas, por medio del equilibrio reflexivo, en una concepción política que pueda servir como eje de un consenso solapado y de esta manera aumentar la estabilidad. $Y$ dada la «imposibilidad práctica» de alcanzar el acuerdo sobre la verdad de las doctrinas generales (PL 63), parece seguirse que tal concepción de la razón pública habría de ser kimparcial (...) entre los puntos de vista de las doctrinas generales razonables» (PL xix). Por tanto, la prosecución de un propósito práctico frente a una imposibilidad práctíca dicta la estrategia teórica de Political Liberalism.

La misma preocupación por el consenso a la luz del hecho del pluralismo razonable determina el alcance de lo que puede considerarse como buenas razones en las deliberaciones públicas sobre cuestiones básicas. «Puesto que muchas doctrinas son consideradas como razonables, aquellos que insisten, cuando hay cuestiones políticas fundamentales sobre la mesa, en lo que ellos consideran verdadero pero otros no, aparecen ante los demás como si únicamente insistieran en sus propias creencias (...) Imponen sus creencias porque, dicen, sus creencias son verdaderas y no porque sean sus creencias. Esto lo puede afirmar todo el mundo; pero también se trata de una afirmación que no puede hacerse valer como buena ante la generalidad de los ciudadanos. Por tanto, cuando hacemos tales afirmaciones, otros que son razonables nos considerarán irrazonables» (PL 61) e incluso «sectarios» (PL 129). Es decir, los agentes políticos que «insisten» en el foro público en aquello que consideran verdadero pero otros no, son «irrazonables». El propósito del actor político de actuar sobre bases públicamente justificables es, de alguna manera, refractado a través del reconocimiento por parte del observador político del hecho del pluralismo razonable, convirtiéndose en una especie de deseo de 
evitar la controversia ideológica acerca de cuestiones fundamentales. En el discurso político esta idea de ser «razonable» desplaza a la de verdad moral. «Dentro de una concepción política de la justicia, no podemos definir la verdad como dada por las creencias defendidas incluso en un consenso idealizado, al margen de lo amplio que sea (...) Una vez aceptamos el hecho de que el pluralismo razonable es una condición permanente de la cultura pública bajo instituciones libres, la idea de lo razonable es más adecuada como parte de la base de una justificación pública» (PL 129). Y esa idea, que funciona más como una versión puesta al día de la idea liberal de tolerancia que como la idea kantiana de razón, sirve para definir también la forma de la justicia internacional.

Al igual que en Political Liberalism, «The Law of Peoples» de Rawls está marcado por una preocupación dominante por la «posibilidad» y «practicabilidad» de los ideales políticos cuando se enfrentan al pluralismo cultural e ideológico. Aquí también «el arte de lo posible» modela la construcción de una teoría de la justicia. Y aquí también el propósito de «estabilidad bien motivada» señala algo más que un requisito funcional del orden social: Rawls quiere asegurarse y asegurarnos que su concepción política de la justicia internacional puede ser el eje de un consenso solapado, porque tal cosa significaría que no es sólo un modus vivendi, sino que podría ganar apoyo razonado (de distintas formas) de agentes libres e iguales en tanto racionales y razonables. No obstante, como ahora argumentaré, las desventajas de esta estrategia para la construcción de ideales políticos arealistas" se hace cada vez más evidente a medida que aumenta el grado de diversidad que ha de ser abarcado.

El espíritu de realismo es evidente en el punto de partida de Rawls: «Los pueblos, en tanto corporaciones organizadas por sus gobiernos, existen de alguna forma en todo el mundo. En términos históricos, todos los principios y patrones propuestos por el derecho de gentes deben, para ser posibles, mostrarse aceptables para la opinión pública considerada y reflexiva de las gentes y de sus gobiernos» (LP 50) ${ }^{13}$. Rawls observa que las fronteras nacionales existentes son «históricamente arbitrarias» (LP 223, n. 16), y ciertamente es consciente de que muchas de ellas se han trazado con sangre; pero evidentemente no piensa que esto las descalifique para jugar el papel fundamental que se les asigna. Es ésta una decisión teórica importante: hacer de algo moralmente arbitrario, como el sistema de naciones, el fundamento de una teoría ideal, y se hace sin justificación formal o «construcción» alguna. Rawls sugiere (LP 223, n. 16) que esto se podría justificar atendiendo a los valores políticos a los que pueden servir los Estados como subunidades de un sistema mayor, pero ignora los problemas específicos de la forma Estado-Nación - las reclamaciones en conflicto de distintos grupos nacionales por el mismo territorio y la presencia de minorías etnoculturales irreconciliables dentro de las fronteras nacionales - así como el hecho de que los Estados-Nación tan sólo cubren el globo terrestre tras la Segunda Guerra Mundial, a menudo siguiendo los patrones de la cartografía colonial, etc. Por otro lado, tampoco presta mucha atención a las múltiples indicaciones de que el sistema del estado-nación, al menos en su forma clásica, bien pudiera estar camino de la obsolescencia. Esto no niega que

\footnotetext{
${ }^{13}$ (The Law of Peopless será citada entre paréntesis en el texto como LP.
} 
los Estados-Nación serán probablemente los actores más importantes en el escenario mundial durante el tiempo venidero, ni que los movimientos de independencia nacional sean todavia los medios principales para lograr la autodeterminación por parte de poblaciones históricamente subyugadas. Pero en este preciso momento de la universalización de la forma Estado-Nación está siendo superada por las realidades de la globalización. Más aún, hay razones teóricas y prácticas para pensar en la justicia cosmopolita en términos posnacionales. Una de las más importantes, como elaboraré más adelante, es simplemente que enfoques como el de Rawls acerca de lo que Kant llamó Volkemecht, el derecho de gentes, hacen «de las gentes y sus gobiernos» las partes a las que los principios de justicia internacional han de mostrarse aceptables ${ }^{14}$, mientras que aproximaciones como la de Habermas a lo que Kant denominó Weltbürgerrecth, derecho cosmopolita, hacen de los ciudadanos individuales (del mundo) el tribunal último de apelación.

El enfoque de Rawls precisa, y él es consciente de esto, que el contenido especificamente liberal-democrático-igualitario de su concepción del liberalismo político sea, en muy último término, considerablemente rebajado. Porque aunque la concepción de la justicia internacional se construyó como una forma de ampliar la concepción liberal de la justicia nacional, el modo de ampliarla es tal que «produce un derecho de gentes más general que no prejuzga a las sociedades no liberales» (LP 65). Por ejemplo, en lugar de insistir en la idea liberal de ciudadanos iguales y libres con determinados derechos básicos, Rawls busca proporcionar una concepción alternativa de los derechos humanos que «no pueda ser rechazada como peculiarmente liberal o propia de nuestra tradición occidental» y que sería en tal sentido "políticamente neutral» (LP 69). De igual forma, al prescindir de las características igualitarias de la justicia como equidad busca alcanzar una descripción de la justicia que tenga la «mayor generalidad» demandada por el escenario internacional (LP 51 y ss.). En suma, puestö que «no puede esperarse razonablemente» que las sociedades no liberales acepten los principios liberales de justicia, incluso una concepción política liberal de la justicia internacional sería irrazonable si insistiera en ellos (LP 75), o más bien, especialmente en el caso de una concepción liberal, porque tal insistencia violaría «el propio principio de tolerancia del liberalismo respecto a otras formas razonables de ordenar la sociedad» (LP 80).

La idea de lo «razonable» parece haber quedado bastante diluida aquí; y, nos asegura Rawls, así es como debe ser: «cuanto más se extiende el alcance de la tolerancia, más se relaja el criterio de lo razonable» (LP 78). Entonces uno no puede por menos que preguntar, ¿qué queda de la idea original de lo razonable, más allá de la tolerancia? En Political Liberalism parece abarcar los desacuerdos que caen dentro del alcance de la carga de la prueba o de la razón y, por tanto, puede esperarse que resulten del uso libre de la razón en condiciones democráticas (PL xvi). Ahora parece abarcar todas las diferencias político-culturales, no importa que sean debidas a cargas específicas de la razón o no, esto es, que sean resultado o no, o puedan deberse al uso libre de la razón. Diré algo más sobre esto más adelante. En cualquier caso, Rawls apela aquí a una categoría intermedia entre

${ }^{14}$ aPor gentes enticndo las personas y sus dependientes vistos como una corporación y organizados a trakés de sus instituciones políticas, que establecen los poderes del Gobiernos (LP 221, n.5). 
lo «completamente razonable», que permite una completa e igual libertad de conciencia y libertad de pensamiento, y lo completamente «irrazonable» que las niega enteramente; esta categoría es lo «no irracional», que admite «una medida», aunque no sea ésta una medida igual y completa como las anteriores (LP 63, 225, n. 28). $\mathbf{Y}$ puesto que las «sociedades jerárquicas bien ordenadas», organizadas a través de religiones estatales, que el derecho de gentes busca expresamente acomodar, caen tipicamente bajo esta categoría, ese derecho estaría mejor caracterizado como propio de una socicdad de pueblos no irrazonables, y su correspondiente consenso solapado sería el que se obtiene entre doctrinas generales no irrazonables. Por tanto, desde $A$ Theory of Justice, pasando por Political Liberalism, hasta «The Law of Peoples», la idea de justicia sufre un debilitamiento progresivo con el fin de acomodar un grado progresivamente mayor de diversidad cultural que Rawls considera teóricamente irreductible.

Permítanme ahora que me concentre en algunos de los detalles del argumento de "The Law of Peoples" y que analice lo que transpiran. Como se ha dicho antes, su estrategia es kextender» la descripción política de la justicia de una sociedad liberal-democrática autosuficiente a una descripción de la justicia de una sociedad de sociedades bien ordenadas, de las cuales sólo algunas son liberales. Esta ampliación tiene lugar en dos «fases» principales: la primera desarrolla una teoría ideal de la justicia, que presume completa aceptación y condiciones favorables; la segunda, la de la teoría no ideal, se ocupa de las cuestiones que surgen de la no aceptación y de las condiciones desfavorables que caracterizan al mundo real. «La teoría no ideal se interroga acerca de cómo puede realizarse la concepción ideal de la sociedad de pueblos bien ordenados, o al menos de cómo se puede trabajar en pos de este objetivo (...) Así concebida, presupone que disponemos de una teoría ideal (...) $\mathrm{Y}$ aunque las condiciones específicas de nuestro mundo cn un tiempo dado - el status quo- no determinan la concepción ideal (...), esas condiciones sí afectan las respuestas a las preguntas de la teoría no ideal [que] son preguntas de transición» (LP 71 y ss.). Los ecos kantianos en la enunciación de su enfoque son inconfundibles; pero, como veremos, Rawls los orienta en una forma francamente antikantiana, de tal modo que las características del status quo son proyectadas en la teoría ideal misma.

En la descripción de Rawls la teoría ideal opera en dos «pasos». Primero, la concepción liberal de la justicia nacional se extiende al derecho de gentes de las sociedades liberales; después es ampliada más aún para incluir las sociedades bien ordenadas no liberales. Ambos pasos utilizan la «posición originaria» como mecanismo de representación, sólo que ahora las partes son representantes de sociedades en lugar de individuos ${ }^{15}$. $\mathrm{Y}$ a cada paso las partes son situadas simé-

${ }^{15}$ Hay, no obstante, un adelgazamiento considerable del kelo de ignorancia» en cl segundo paso. Los representantes de regímenes jerárquicos bien ordenados conocen qué sociedades $y$, por tanto, qué intereses representan: *Los intereses de cada sociedad jerárquica son entendidos por sus representantes de acuerdo con su concepción de la justicia o son presupuestos por ellas (LP 64). Esto parece senalar un cambio con la construcción paralela del derecho de las naciones en $A$ Theory of Justice (véase especialmente la p. 378). En cualquier caso, aquí la posición original ciertamente modela una concepción muy diferente de la razón política práctica, a diferencia de A Theory of Justice o Political Liberalism. 
tricamente, de forma que se modelen condiciones imparciales de deliberación entre los representantes de los pueblos, los cuales, en tanto pueblos, se consideran a sí mismos como libres e iguales. Por último, es, dice Rawis, «fundamental» para su descripción del derecho de gentes que «tanto las sociedades liberales como las jerárquicas», así representadas en tanto situadas razonablemente y racionales, «lo acepten» (LP 52). Esto es lo crucial en este asunto: la estrategia de Rawls requiere que la concepción política ideal misma de la justicia internacional sea aceptable para todos los pueblos «bien ordenados», tanto si sus propias concepciones de la justicia son o no liberales, democráticas o igualitarias. De esto se sigue que centrarse en un consenso solapado alcanzable entre pueblos liberales y no liberales, democráticos y no democráticos, igualitarios y no igualitarios, puede no ser específtcamente liberal, democrático e igualitario. ¿Pero por qué ha de denominarse ideal al común denominador resultante de tal estrategia? ¿Y por qué cree Rawls que resultará aceptable para sociedades que están organizadas de acuerdo con principios liberales, democráticos o igualitarios? ¿Por qué han de obviar sus principios políticos básicos en favor de alcanzar un acuerdo político con pueblos que no los comparten? Porque al contrario que el consenso solapado en el caso nacional, no sólo son dejadas a un lado las diferencias en las doctrinas generales, sino también diferencias clave en las concepciones políticas de la justicia.

Rawls trata de reducir esta tensión estrechando el abismo entre ambas partes: a) construyendo dentro de su concepción de las sociedades jerárquicas bien ordenadas elementos que cree que los liberales considerarán como los requisitos mínimos de la decencia política (LP 69), y b) dejando caer de su concepción del derecho de gentes para sociedades liberales (el primer paso de su teoría ideal) elementos que crec que algunas socicdades jerárquicas considerarán objetables.

a) Las sociedades jerárquicas bien ordenadas están caracterizadas por: 1) ser pacíficas y no expansionistas; 2) estar informadas por una buena concepción común de la justicia, sostenida por una jerarquía razonable de expertos, de forma que los regímenes sean legítimos a los ojos de sus propios pueblos, y 3) por ser respetuosas con los derechos humanos básicos.

b) El derecho de gentes para una sociedad de sociedades liberales no incluye las características igualitarias de la equidad -esto es, el valor imparcial de las libertades políticas, la equitativa igualdad de oportunidades y el principio de la diferencia - puesto que ya se ha dicho que son inaceptables para otras sociedades liberales informadas por otras concepciones de la justicia menos igualitarias.

Thomas Pogge ha cuestionado con efectividad lo acertado de esta degradación de la preocupación por la igualdad en la teoría internacional de la justicia ${ }^{16}$. No

\footnotetext{
Por tanto, la conjetura de Rawls de que una construcción que arranque de una posición original absolutamente inclusiva, con representantes de todas las personas individuales del mundo, llegaría al mismo derecho de gentes (LP 66) es, como poco, altamente implausible. Al igual que la posición originaria en trabajos anteriores, ésta habría de modelar una concepción liberal de de las personas como libres e iguales y conducir, parece, a una concepción de la justicia internacional mucho más cercana a una teoría cosmopolita del Derecho.

${ }^{16}$ Thomas W. Pogge, «An cgalitarian Law of Peoplesw, Philosophy and Public Affairs 23 (1994), pp. $[95-224$
} 
voy a revisar este artículo aqui. Pero me gustaría hacer la observación general de que un enfoque de la justicia que considera la libertad individual, la participación política y la justicia social como teóricamente interdependientes no puede desprenderse coherentemente de una de estas preocupaciones y retener las otras. En este punto, los argumentos de Rawls a favor de esta concepción igualitaria de la justicia nacional pueden ser usados contra su concepción no igualitaria de la justicia internacional. Como en el caso nacional, las desigualdades sociales y económicas afectan no sólo las oportunidades de los individuos y de los colectivos de influir las decisiones políticas que establecen los términos de la cooperación social, sino también sus oportunidades de modelar sus propias vidas de acuerdo con sus propias concepciones de lo bueno. Recuérdese que la justicia social excluye las desigualdades sociales y económicas que sean tan grandes que conviertan las libertades básicas en «meramente formales» (PL 325). Requiere mantener el «valor de equidad» de las libertades políticas y maximizar los bienes primarios (medios para todo uso) disponibles por los miembros menos aventajados de la sociedad. La razón del primer requisito es que sin acceso igual de imparcial al proceso político kel trasfondo de instituciones justas es prácticamente imposible que sea establecido o mantenido» (PL 328). La razón de este último es que el *valor» 0 «utilidad» reales para las personas de sus derechos y oportunidades garantizados institucionalmente depende críticamente de los medios a su disposición para ejercerlos o beneficiarse de ellos (PL 325).

En una serie posterior de conferencias, no publicadas, sobre «Los problemas filosóficos del derecho de gentes», Rawls se ocupa de esta cuestión, pero arguye contra la incorporación del principio de justicia distributiva global en el caso ideal, y defiende, por contra, un «deber de asistencia» en el caso no ideal de sociedades sobrecargadas de condiciones desfavorables ${ }^{17}$. El propósito a largo plazo es que cada sociedad devenga - si es necesario con la ayuda de sociedades en mejores condiciones- un «miembro libre y autosuficente en la sociedad de pueblos y por tanto que sea capaz de gestionar su vida política de forma razonable y racional..." ${ }^{18}$. Una vez se logra esto se presupone que cada sociedad se ocupará del bienestar de sus propios miembros. Pero este argumento sólo reproduce muchas de las dificultades de los individuos abstractos del liberalismo clásico en el nivel igualmente abstracto de las sociedades autónomas. Ignora, en particular, la conexión entre el poder socioeconómico desigual y el desigual poder político $y$, por tanto, pone en riesgo «el imparcial valor de las libertades políticas» en la arena internacional. Quizá una razón por la que esta objeción familiar no aparece en la discusión de Rawls es que su derecho de gentes no sólo rebaja las preocupaciones distributivas sino las democráticas, como ahora paso a explicar.

La tesis central de la entera construcción de Rawls, a saber, que las sociedades bien ordenadas no liberales aaceptarán» el mismo derecho de gentes que las socie-

${ }^{17}$ Me refieto al manuscrito mecanografiado que sirvió de base para los tres seminarios que Rawls impartió en la Universidad de Princeton en abril de 1995. La discusión sobre la justicia distributiva, que tiene la intención de ser una respuesta al articulo de Pogge citado en la nota 16, aparece en las pp. 75-88 de este manuscrito.

Ibid., pp. 75 y ss. 
dades liberales bien ordenadas (LP 43), gana la plausibilidad que tiene sólo mediante el recurso de dejar caer silenciosamente, o de empujar al trasfondo del derecho de gentes para sociedades liberales (esto es, el primer paso en la teoría ideal) una fuente potencial de desacuerdos fundamentales con las sociedades no liberales, a saber, los elementos democráticos de la justicia como imparcialidad. Su bosquejo de los «principios de justicia entre pueblos iguales y democráticos» omite sorprendentemente cualquier mención a instituciones y procesos específicamente democráticos (LP 51-59). Simplemente no se discuten en este paso, de forma que no son un problema en el siguiente, cuando los pueblos no democrăticos aparecen en escena. "En las sociedades democráticas», observa Rawls, «las personas serán ciudadanos, mientras que en las sociedades jerárquicas y en otras serán miembros» (LP 221, n. 5). Y los derechos de tales miembros, deja claro, no necesitan incluir el cabal complemento de libertades políticas para que sus sociedades disfruten de plena pertenencia a la sociedad de pueblos. Lo que hace esta separación de las libertades personales de las políticas es, en efecto, dar la vuelta a la transformación democrática, ocurrida en los siglos $\mathrm{xIX}$ y $\mathrm{xx}$, de los derechos civiles conferidos anteriormente por el Estado absolutista. Y ese movimiento es incompatible con las relaciones internas entre lo que Rawls denomina slas libertades de los modernos y las libertades de los antiguos». En su repuesta a Habermas, por ejemplo, se empeña en demostrar que la justicia como imparcialidad reconoce «la conexión interna o la mutua presuposición» entre la «autonomía privada y la pública» ${ }^{19}$. Pero en «The Law of Peoples» las separa, y deja el concreto significado de los dercchos humanos, incluyendo la especificación de los aspectos en los cuales los miembros han de ser tratados como iguales ante la ley, a la determinación de las autoridades, sin la participación democrática o la representación de aquellos sujetos a las mismas. Rawls requicre que scan representados, en tanto miembros de grupos, en lo que llama «una jerarquía consultiva razonable» (LP 61 y ss.). Pero esto es hacer retroceder varios siglos el reloj de nuestra comprensión de la interpretación y cumplimiento de los derechos básicos.

Al desprenderse explícitamente de las características de la justicia como imparcialidad y al marginar sus rasgos democráticos, la tesis central de Rawls deviene algo más plausible - algo más, pero no enteramente-. Todavía ha de ocuparse de la circunstancia de que las sociedades jerárquicas bien ordenadas en torno a religiones estatales aceptarán sólo un sistema restringido de derechos individuales básicos si lo comparamos con el complemento habitual liberal. En consecuencia, Rawls requiere que las sociedades jerárquicas bien ordenadas tan sólo respeten los «derechos mínimos» a la vida, a la libertad, a la propiedad personal, la igualdad ante la ley y la emigración (LP 62). No se les requiere que aseguren la completa libertad de conciencia y de libertad de pensamiento, sino sólo una "porción» de ambas (LP 63); ni necesitan permitir la libertad de expresión como en las sociedades liberales - todo lo cual tiene, por supuesto, importantes implicaciones no sólo

${ }^{18}$ John Rawls, «Reply to Habermas», Joumal of Philosophy, 92 (1995), pp. 132-180, en la p. 153. Rexpondiendo a Jürgen Habcrmas, «Reconciliation through the Public Use of Reason: Remarks on John Rawls's Political Liberalism», tr. C. Cromin, en el mismo número, pp.109-131. 
para el uso de la razón pública en tales sociedades, sino también para la «razonabilidad» de los acuerdos y desacuerdos modelados por ella.

La ventaja estratégica de concebir los derechos humanos de esta manera, de acuerdo con Rawls, es que «no dependen de ninguna doctrina moral general particular (...), tal como, por ejemplo, que los seres humanos son personas morales que tienen igual valor»; porque tal doctrina es una doctrina que muchas sociedades jerárquicas spodrian rechazar como liberal o democrática, o de alguna manera como distintiva de la tradición filosófica occidental y pcrjudicial para otras culturas", mientras que su propia estrategia se dirige a identificar requisitos «políticamente neutrales» para un «régimen mínimamente decente» (LP 69). Esta es, sostiene, todavía una estrategia liberal, porque empieza en el liberalismo político y «lo extiende» al derecho de gentes; pero no está dirigida a la contrucción de una concepción distintivamente liberal de la justicia internacional ${ }^{20}$. Por el contrario, muestra alos límites de la tolerancia» para encontrar «el lecho de roca más allá del cual no podemos ( inosotros los liberales?) ir» (LP 78 y ss.).

Habría mucho sobre lo que detenerse en este boceto de derecho de gentes. No me ocuparé en lo que sigue de los detalles, ni siquiera me detendré en detalles no menores, como la disminución radical de derechos civiles, sociales y políticos. En su lugar desearía concentrarme en la estrategia teórica que adopta Rawls. Tanto su terminología como sus referencias sugieren que está revisando de forma consciente la estrategia kantiana para encajarla de la mejor manera posible en una comprensión destrascendentalizada de la razón. Kant todavía funcionaba con la distinción clásica del racionalismo entre convicción y persuasión ${ }^{21}$. Sostener que algo es verdad se decía que era convicción si descansaba en sfundamentos objetivos» y era, por tanto, «válido para todo ser que posea razón». Se decía que era persuasión si sus fundamentos se basaban sólo «en la indole cspecial del sujeto».

2h Tal como han argumentado Samuel Scheffler [sThe Appeal of Political Liberalism», Ethics, 105 (1994):4-22] y Leif Wenar [kPolitical Liberalism: An Internal Critiques Ethics, 106 (1995), pp. 32-62], la estrategia neutralista de Rawls falla incluso en el caso nacional, puesto que su presentación de la concepción política descansa sobre ideas (por ejemplo, la carga del juicio, la concepción de la persona, el método del constructivismo político mismo) que no todas las partes del consenso solapado podría cspcrarse razonablemente que aceptaran. El problema es exacerbado en el contexto presente, en el que la concepción política del đerecho de gentes es explícitamente presentada como una "extension" del liberalismo politico. Aqui, la «esperanza» (LP 230, n, 54) de Rawls de que las sociedades jerárquicas bien ordenadas puedan abrazarlo -que sirva como foco de un consenso solapado en el terreno internacional - tiene una base bien diferente de la que tiene la esperanza correspondiente para su concepción del libcralismo político en el caso nacional. La esperanza de que la última se muestre públicamente aceptable estaba fundada en el método mismo de su construcción, que comenzaba con «ideas fundamentales implícitamente compartidas en la cultura política pública» de una (nuestra) sociedad liberal (PL 100). La esperanza correspondiente en un derccho de gentes significa unificar diferentes culturas políticas que no tienen tal fundamento. A este respecto, los esfuerzos "prácticos" de Rawls por proporcionar una concepción «realista» de la justicia internacional no cierran el abismo entre lo ideal y lo real, ni mucho menos en la medida en la que él evidentemente lo desea. En concordancia, los costes comparativos de volver a un enfoque más estrictamente universalista no son tan altos a este respecto como pareciera a primera vista.

${ }^{26}$ Véase, por ejemplo, la Crítica de la razón pura, A820-1/B848-9, de la que están tomadas las palabras citadas en las frases siguientes. 
Pero Kant también reconoció que en la práctica el «criterio» mediante el que determinamos si sostener algo como verdadero es una cosa u otra es sla posibilidad de comunicarlo y comprobar su validez para toda razón humana». Es sólo en el esfuerzo, y a través de éste, por asegurar un acuerdo universal, escribió, como podemos realizar el «ensayo (...) de ver si producen en el entendimiento de otros el mismo efecto que en el nuestro». Sin embargo, la desublimación de nuestra comprensión de la razón y de la racionalidad en los dos siglos transcurridos desde que propuso estas opiniones significa que algunos de los factores que consideró como «subjetivos» nosotros los consideramos como inevitablemente presentes en los procesos de comunicación racional orientados a alcanzar acuerdos razonados. $\mathrm{Y}$ esto hace necesario equilibrar su acento sobre la unidad sistemática con un reconocimiento correlativo de la diversidad ineliminable.

Es esto, entre otras cosas, lo que Rawls intenta hacer con sus nociones de carga de la razón, pluralismo razonable y consenso solapado. Pero su manera de hacerlo diluye tanto la idea de un acuerdo razonable que la idea correspondiente de validez objetiva pierde mucho de su idealidad trascendente de la realidad. Esto ocurre, en gran medida, porque el pluralismo razonable que él esperaría surgiera del aejercicio de la razón humana en instituciones libres» (LP 82) cs, en su construcción de una concepción política de la justicia, tácitamente reemplazado por un pluralismo de facto de doctrinas generales que satisface los mucho más débiles requisitos establecidos por su noción revisada de tolerancia. Esto significa que en la construcción de Rawls, por comparación con la de Kant, las ideas de ilustración y crítica juegan un papel marginal. Su teoría «libremente flotante» de la justicia no toma posición respecto a las demandas de validez que no estén directamente en relación con el contenido de la concepción política tal como él la entiende.

Esto queda claro en la «segunda fase» de su bosquejo de un derecho de gentes, el de la teoría no ideal, que se ocupa de cómo puede ser desarrollada la concepción ideal en las circunstancias no ideales del mundo real. Las condiciones desfavorables que toma en consideración incluyen deficiencias no sólo en los recursos materiales y tecnológicos, y en capital humano y conocimiento experto, sino también en las tradiciones políticas y culturales. «Muchas sociedades con condiciones desfavorables to carecen de recursos (...) Por el contrario, el problema es por lo común la naturaleza de la cultura política pública y las tradiciones religiosas y filosóficas que subyacen a sus instituciones» (LP 77). Si el interés general que hay tras la teoría no ideal es ayudar a sociedades «ahora apesadumbradas por condiciones desfavorables (...) en pos de condiciones que hagan posible una sociedad bien ordenada» (LP 75), parecería seguirse que entre sus preocupaciones principales cstaría la liberación de tales culturas políticas públicas y la crítica de tales tradiciones, esto es, algo muy parecido a lo que Kant entendía por ilustración. Pero Rawis no toma este camino, que desde su perspectiva equivale a una nueva variante de etnocentrismo. Por el contrario, caracteriza el problema de la cultura política en términos tan «políticamente neutrales» como «gobierno opresivo» y kelites corruptas» (LP 77), y esto hace compatible, presumiblemente, el deber de promover condiciones político-culturales favorables con la evitación del etnocentrismo - con una interesante excepción: «la subyugación de las mujeres incitada por religiones 
no razonables» es señalada como una condición desfavorable que debemos cambiar (LP 77) - Pero si Rawls está deseoso de tolerar este pedazo no insignificante de crítica ilustrada, no está claro sobre qué base puede oponer críticas a la «religión irrazonable» y a otras doctrinas generales que justifican el tratamiento desigual de otros grupos identificados por raza, etnicidad, sexualidad, clase, estatus, casta u otro marcador -especialmente, puesto que es difícil de ver que las cargas de la razón, consideradas estrictamente, sean lo suficientemente amplias como para tolerar aquellos elementos de las cosmovisiones recibidas que reclaman garantizar tal subordinación.

De manera más general, el método de Rawls de evitación elimina cualquier justificación en teoría para criticar las doctrinas generales que sirven para apoyar estructuras sociales y políticas jerárquicas. Así corre inconscientemente el riesgo de situar la teoría ideal en el lado de los poderes fácticos y de las elites gobernantes reales, siempre que no sean opresivas y corruptas, y de privar de medios de apoyo a movimientos indigenas de liberación, democratización, justicia social, que también son parte del mundo real. Si un régimen establecido satisface sus tres requisitos de buena ordenación, no hay nada que su ideal de justicia política pueda ofrecer a aquellos que buscan cambiar la realidad de condiciones iliberales, antidemocráticas o desiguales, nada a lo que puedan apelar los movimientos de reforma y las luchas de liberación para justificar completa libertad de expresión, pensamiento y conciencia, y mucho menos completa igualdad.

¿Qué ha ido mal aquí? Hablando en general, la forma de Rawls de respetar la diferencia cultural le deja muy cerca de uno de los extremos del espectro que va desde la tolerancia y el acomodamiento a la crítica y la transformación: construye demasiadas diferencias de facto en su idea de pluralismo razonable. El destripamiento del contenido normativo de tal idea desplaza la neutralidad liberal demasiado cerca de la aquiescencia política conservadora. Esto, me parece, es en gran medida consecuencia de su ampliación del círculo de la tolerancia hasta incluir diferencias político-culturales fundamentales. En Political Liberalism el pluralismo razonable encuentra sus límites en el consenso solapado sobre la concepción política de la justicia. Las raíces de tal consenso se encuentran precisamente en las ideas y valores implícitos y compartidos de una cultura política común que proporcionaba los matcriales con los que se construyó la concepción de la justicia mediante el método del equilibrio reflexivo. Esta era la única manera, argumentaba Rawls, para que la teoría política lograra su tarea principal, a saber, encontrar un acuerdo político-legal básico en medio de la diversidad cultural e ideológica. Pero a nivel global, la prosecución de la misma tarea mediante el mismo método se enfrenta con el formidable obstáculo de la extrema diversidad de culturas políticas. La dccisión de Rawls de buscar un terreno común en medio de tal diversidad está tras su enfoque de común denominador en el derecho de gentes. Y esto conduce, como es natural, a reducir el número y a diluir el contenido de libertades civiles básicas, a aceptar la consulta de jerarquías como un sustituto de la autodeterminación democrâtica y a colorear la justicia distributiva en términos de concepciones de la justicia del wbien común». Sin duda se pueden reemplazar estos movimientos específicos con otros, pero la estrategia misma, me parece, requeriría equivalentes funcionales. 
Esto es en parte debido a la tendencia de Rawls a ver las culturas políticas nacionales como si estuvieran hechas de un único paño. Asi, las diferencias entre la liberal y la no liberal son representadas en términos de «nosotros» frente a aellos". Subraya, por ejemplo, que aquellos que tienen puntos de vista liberales podrían decir que el mundo sería un lugar mejor si todas las sociedades fueran liberales. «Pero esa opinión (...) pudiera no tener fuerza operativa debido a que, en justicia, lo que ellos, "es decir, nosotros" pueden hacer políticamente (...) "para" afirmar la superioridad de su punto de vista comprensivo particular, es completamente compatible con afirmar una concepción política de la justicia que no la imponga» (LP 81). Esta observación está evidentemente dirigida a los de "fuera»; ignora los desacuerdos internos acerca de tales materias, desacuerdos que caracterizan a nuestras sociedades presentes. Pero aun si, en beneficio del argumento, aceptáramos este esquema nosotros/ellos, ¿los teóricos políticos tendrían que escoger entre la versión de la «neutralidad política» de Rawls y la «imposición» de puntos de vista liberales sobre sociedades no liberales? ¿No hay alternativa compatible con el reconocimiento de las cargas de la razón y la ineliminabilidad del pluralismo razonable?

\section{II}

Un intento por construir tal alternativa podría comenzar mediante la recolocación del límite entre la teoría ideal y la no ideal mucho más cerca de lo que Kant entendía como idea(le)s de la razón. En esta estrategia teórica aunque las cargas de la razón y la ineliminabilidad de desacuerdos razonables habrían de ser integrados en la teoría ideal, porque son rasgos de la razón postmetafísica y postrascendental misma, la realidad de regímenes no liberales, no democráticos y no igualitarios, incluso aquellos que satisfacen los tres requisitos de Rawls de las sociedades bien ordenadas, serían preocupación no de la teoría ideal sino de la no ideal. La teoría ideal podría entonces construir una idea no diluida de un orden internacional liberal-democrático-igualitario que sirviera como patrón final de justicia e injusticia en ese dominio.

Por supuesto, el revisar la comprensión de Kant de la razón afectaría tal construcción. Si siguiéramos la iniciativa de Habermas, por ejemplo, cambiaríamos el eje de la crítica de la razón desde formas de conciencia trascendental a formas de interacción comunicativa. El proyecto ilustrado podría dedicarse entonces al cultivo de formas apropiadas de discurso teórico y práctico y al establecimiento de instituciones y procedimientos que les dieran encarnadura social. Respecto a este discurso práctico, éste incluiría cl criticar y reformar las condiciones que impiden la completa discusión pública de cuestiones legales y políticas entre ciudadanos libres e iguales. Esta estrategia, por supuesto, puede producir una idea de la justicia que sea desvergonzadamente liberal, democrática e igualitaria, y por tanto, desde el punto de vista de Rawls, el peligro de ser etnocéntrica e intolerante. Pero si «ctnocéntrico" significa aquí, como parece, nada más que desarrollar y defender una teoría de la justicia desde un punto de vista que es el propio, entonces tendremos 
que ser, en expresión de Rorty, francamente etnocentristas. Esto tiene la ventaja de dejar a los participantes mismos la tarea sin fin de encontrar un terreno común en lugar de reservársela a esos teóricos más allá del bien y del mal del consenso solapado. Puesto que no hay nada uirracional» en el proponer y defender puntos de vista que uno considera bien fundados, no estamos forzados a elegir entre el etnocentrismo y la tolerancia en el sentido de Rawls.

El cambio clave se da aquí en nuestra concepción de la tarea del teórico. Rawls considera que esta tarea consiste en desvelar y articular bases de acuerdo, en la esperanza de construir una concepción de la justicia internacional que pueda ser el eje de un consenso solapado entre las sociedades politicas existentes. Habermas tiene una concepción más crítica de la tarea de la teoría, más próxima a Kant: la concibe como la articulación de ideas e ideales de la razón que puedan servir como patrones y gulas «en un proceso de ilustración [en el que] sólo pucde haber participantes» ${ }^{22}$. Esta perspectiva, con sus muchas variaciones de los siglos XIX $\mathrm{y} \mathrm{xx}$, hace al teórico, como tal, crítico también de la sinrazón existente y abogado de determinados fines políticos que pueden ser desarrollados con nuestro esfuerzo ${ }^{23}$. Desde este punto de vista, el «acuerdo razonable» no es la descripción de un estado de hechos presente, sino el objetivo de una tarea práctica; no es «desvclado» sino algo que se logra de forma continua. De este modo, caracterizar una concepción de la justicia como universalmente aceptable es una hipótesis práctica: una pretensión al efecto de que todos los «seres racionales», como diría Kant, pudieran ser racionalmente persuadidos de aceptarla.

Este enfoque puede acomodar fácilmente el desacuerdo razonable y el pluralismo razonable. Pero, ¿qué hay del pluralismo de facto de las culturas políticas que Rawls busca acomodar, dentro de determinados límites de «decencia mínima», en su teoría ideal? Si dejamos a la teoría no ideal la pregunta de cómo relacionar los regímenes organizados alrededor de concepciones de la justicia fundamentalmente diferentes de la concepción ideal, entonces no hay necesidad de intolerancia o de imposición. Ésta es una cuestión pragmática, contextual, que no tiene una respuesta fija. Al considerarla debemos tener siempre en cuenta las circunstancias, tendencias, probabilidades y posibilidades contingentes y cambiantes. A menudo, en primer término, se trata de una cuestión que las propias minorías oprimidas (y en ocasiones las mayorias oprimidas) han de sopesar en sus luchas por el reconocimiento social, cułtural, legal y político ${ }^{24}$.

Concluyamos, por tanto, con una reconsideraciôn del ideal cosmopolita a la luz de mis críticas a la versión de Rawls del derecho de gentes. ¿Cómo podemos

${ }^{22}$ Jürgen Habermas, "Some Difficulties in the Attempt to Link Theory and Practices, en Habermas, Theory and Practice (Boston: Beacon Press, 1973), pp. 1-40, la cita en la p. 40.

2. Las avariaciones» más importantes ticnen que ver con el desarrollo de investigación empírica histórica y social en los dos siglos posteriores a Kant. La versión de Habermas de la ateoría criticas toma esto en cuenta al buscar combinar los métodos conceptuales reconstructivos con métodos empirico-explicativos, y aś ir más allá del puro prescriptivismo caracteristico de los enfoques neokantianos. Véanse los capítulos 2 y 8 de Between Facts and Norms para las observaciones sobre la relación entre teorías filosóficas y teorías sociologicas del Derecho y la democracia.

${ }^{24}$ Véase Axel Honneth, The Struggle for Recognition: The Moral Grammar of Social Conflicts (Cambridge, Mass: The MIT Press, 1996). 
pensar la unidad en la diferencia a nivel global pero no en la forma que él lo hace? Hay una cosa clara: tenemos que ir más allá de la actitud rígida de oponer lo universal a lo local o lo local a lo universal. Aunque sea necesario subrayar las diferencias culturales como antídoto frente a los acompańamientos ideológicos de la globalización neocolonial, la mera celebración de la diversidad no enfrenta de forma adecuada la necesidad de unidad global. Ni toma suficientemente en cuenta la miriada de formas en las que la diferencia ha sido enlazada con la falta de libertad, la desigualdad y la opresión. Las diferencias, como sabemos, están construidas relacionalmente, y demasiado a menudo las relaciones en cuestión han sido de dominación y subordinación ${ }^{25}$. Por tanto, la diversidad - tanto a través de los ejes del género, la raza, la etnicidad, la cultura, la sexualidad, la clase, el estatus o lo que sea- ha de ser interpelada críticamente tanto como la unidad. Ninguna es per se legitima o ilegítima; ambas son necesarias; cada una de ellas ha de ser repetidamente reconfigurada en relación a la otra. Más aún, el libre juego de la identidad y la diferencia requiere una amplia estructura de igual respeto, y esto significa no sólo iguales libertades individuales, sino también, de forma interdependiente, igual voz a la hora de definir los términos de la cooperación social e igual oportunidad para desarrollar las capacidades propias y buscar la concepción propia del bien. Por tanto, las politicas de la identidad y de la diferencia deben ir de la mano con las políticas de los derechos, la representación y la redistribución ${ }^{26}$.

He argumentado que el enfoque de Rawls de búsqueda de un terreno común para la concepción internacional de la justicia no es la forma correcta de pensar acerca de tal estructura o tal política. Y ello porque concede demasiada «facticidad» a expensas de la «validez». Esto es, disminuye de manera alarmante los fundamentos normativos de la crítica social. En último término parece deseoso de poner fuera de juego, como prejuicios hacia las culturas no liberales, hasta el axioma básico liberal del igual valor de todos los seres humanos. Y consecuentemente, no propone una concepción del derecho de los ciudadanos del mundo (Weltbürgerrecht, en términos de Kant), sino una versión del derecho de gentes (Vôlkerrecht), de forma que la relación de los individuos con el Derecho internacional está mediada a través de los respectivos «pueblos», considerados como corporaciones organizadas a través de instituciones políticas. Es decir, los ciudadanos no son de forma inmediata ciudadanos portadores de derechos en esta sociedad mundial de sociedades. Esta especie de holismo político no sólo se desprende de la concepción liberal de la ciudadanía, sino que esencializa las diferencias político-culturales -como si los miembros de cada sociedad pensaran todos igual ${ }^{27}$. $6 \mathrm{Y}$ qué ocurre con las divisiones internas y los desacuerdos? ¿Qué ocurre con las comunidades más pequeñas

\footnotetext{
3 Véasse Iris Young, Iustice and the Politics of Difference (Princeton University Press, 1990).

25 Nancy Fraser ha desarrollado una argumentación sólida de la conexión entre las políticas del reconocimicnto con las politicas de la redistribución en aMulticulturalism and Gender Equity: The U. $S$. Difference Debates Revisited», Constellations, 3 (1996). pp. 61-72.

"Este holismo esencializador es integrado en la concepción de Rawis de la posición original en el segundo paso del primer estadio: los pueblos o sociedades son los representados y no los individuos - los subgrupos. Esto asume un grado de unanimidad intema que es en exiremo irreal. Para que las minorías, los grupos disidentes, los movinientos de reforma, etc., no se queden sin voz a la hora de
} 
que siempre hay dentro de colectivades mayores? ¿Qué ocurre con los grupos subordinados que luchan contra el gobierno de elites dominantes? ¿Qué pasa, en particular, con los grupos que promueven cambios liberales, democráticos o igualitarios en las sociedades jerárquicas?

Teniendo presentes estas dificultades y las otras discutidas antes, tornémonos ahora a considerar la ruta alternativa que desde la «Paz Perpetua» de Kant ha tomado Jürgen Habermas. En lugar de presentar una descripción de sus opiniones sobre la justicia internacional, aquí me ocuparé meramente de algunos de los requisitos que suscita a este tipo de universalismo sensible al contexto el tomarse en serio la respuesta a la preocupación de Rawls por el pluralismo, sobre todo si, al mismo tiempo, queremos evitar lo que he argumentado son las debilidades de la concepción de este último ${ }^{28}$.

1. En línea con mi crítica al colectivismo inherente al enfoque völkerrechtich, una concepción alternativa weltbürgerrechtlich de la justicia internacional habria de incluir un programa de derechos ( «humanos») básicos de los ciudadanos del mundo y procedimientos legalmente institucionalizados para su interpretación, implementación y cumplimiento. Esto significa, ciertamente, que la soberanía local ha de ser relativizada cn cste respecto. $Y$ así ha de ser. Una teoría de la justicia internacional debe apelar más allá de los patrones locales si es que ha de tener algún mordiente normativo o crítico. El teórico político ha de reconocer simplemente que no puede haber ganancia sin pérdida, y que uno no puede desafiar lo modelos establecidos de autoridad sin desafiar las creencias y valores establecidos.

2. Pero un derecho cosmopolita dirigido, en primer lugar, a los individuos como ciudadanos del mundo no necesita excluir el reconocimiento de comunidades políticas no globales. La incorporación por capas múltiples de los ciudadanos es una realidad ya familiar. Muchos de nosotros formamos parte simultáneamente con derechos y responsabilidades especiales de municipios, condados, Estados, provincias, naciones $\mathrm{e}$ incluso uniones políticas supranacionales. $\mathrm{Y}$ no hay buenas bases conceptuales en contra de expandir tal sistema de pertenencia paralela al nivel global. Sin embargo, los modelos de confederación difusa propuestos por Kant y Rawls e implementados en cierta medida en la Liga de Naciones y en las Naciones Unidas se han mostrado demasiado débiles como para establecer un gobierno efectivo del Derecho internacional. For otra parte, la advertencia de Kant de que un Leviatán global daría lugar o a la opresion global o a la guerra civil global ha sido repetida, con buenas razones, por la mayoría de los teóricos

establecer los términos de la cooperación internacional, cada sociedad habrá de tener una pluralidad de representantes, uno de cada subgrupo con creencias, valores e intereses distintos de forma relevante. No es difficil imaginar que esta línea de pensamiento pudiera reconducir en últimn término al esquema de una persona-un representante de otras obras de Rawls.

24 Tal como entiendo a Habermas, estos requisitos son compatibles con sus opiniones: de hecho, se ha ocupado de la mayoría de ellos. La principal divergencia viene en el punto 6 y concierne al papel que asigna al consenso racionalmente motivado en su construcción de la legitimidad política. Sobre este punto véase Thomas McCarthy, wegitimacy and Diversity: Dialectical Reflections on Analytical Distinctions", Cardozo Law Review, 17 (1996). pp. 1083-1125, y la «Róplicam de Habermas, en el mismo número, pp. 1477-1557, especialmente las pp. 1487-1503. 
de las relaciones internacionales desde entonces. Pero debería parecer obvio que estas dos alternativas no agotan el espectro de posibilidades institucionales, que entre ellas hay un campo fértil en el que concebir muchos tipos diferentes de confederación, desde las federaciones de Estados-Nación, cuya soberanía es limitada por un Estado de Derecho internacional, a las federaciones que incorporan no sólo comunidades políticas territorialmente definidas, sino también comunidades no localizadas - por ejemplo, en diáspora-. Qué tipos sean posibles y deseables bajo qué circunstancias es, claramente, algo que no ha de determinarse sólo por la teoría. Lo que puede decirse a este nivel general es que el modelo cosmopolita de miembros por capas o paralelos requiere que los modos locales de incorporación estén sujetos a las constricciones de derechos, garantías y protecciones globales. Por otra parte, tal como es concebido en este modelo, las unidades políticas subglobales son algo más que simples subunidades del Gobierno mundial: tienen su propia presencia moral precisamente como organizaciones legales-politicas en las cuales los miembros se asocian libremente en términos aceptables para todos, y en las que los derechos y obligaciones de los miembros son definidos y garantizados. Habrá siempre, parece, una necesidad de unidades de gobierno menores que la global, y todos lo Gobiernos legítimos merecen respeto como expresión de las voluntades de sus pueblos y como garantes de sus derechos básicos.

3. Dada la interdependencia entre los Derechos civiles, políticos y sociales, el modelo de una federación de unidades políticas subglobales constreñidas por una única estructura cosmopolítica demanda no sólo un sistema de tribunales que interpreten y apliquen el Derecho internacional, sino también instituciones y procedimientos globales para claborarlo, implementarlo y hacerlo cumplir, incluyendo mecanismos de dirección y redistribución que se ocupen de las disfunciones y de las desigualdades de la economía global de mercado. La necesidad de lo recién enunciado me parece teóricamente clara; los medios prácticos para lograrlo, sin embargo, cualquier cosa menos eso. Es evidente, en la historia de los Estados constitucionales, que si los derechos no han de ser conferidos, definidos e implementados meramente de forma paternalista, es indispensable alguna forma de participación, representación y responsabilidad democrática, y para que estén al alcance de todos los ciudadanos los recursos materiales para el ejercicio de los derechos formales y para el disfrute de una vida decente, han de intervenirse los mercados y han de corregirse las grandes dislocaciones a las que dan habitualmente lugar. Pero esta historia también deja claro lo dificil que es conseguir la autodeterminación democrática y la justicia social en sociedades complejas nacionales, y no digamos en la sociedad planetaria. Aquí no puedo hacer otra cosa que reconocer estas dificultades y remitirme a la creciente literatura dedicada al asunto ${ }^{29}$.

4. El problema del que me he venido ocupando aquí es de naturaleza más conceptual, a saber, cómo pensar la unidad de una estructura legal y política global en la diversidad de las culturas locales. Un universalismo multicultural debería,

${ }^{2}$ Dos publicaciones recientes con discusiones interesantes sobre la democracia y la justicia social globales son, Jespectivamente, las de David Held, Democracy and the Global Order (Oxford: Polity Press, 1995), y Martha Nussbaum y Amartya Sen, eds., The Quality of Life (Oxford, Clarendon Press, 1993). 
al menos, dejar de hablar de la modernidad en singular y hacerlo en su lugar de «modernidades alternativas» ${ }^{30}$. Igualmente, habría de admitir la diversidad dc realizaciones de la libertad individual, de la democracia política y de la justicia social, todas constreñidas, sin embargo, por una estructura abstracta de derechos humanos básicos legalmente institucionalizados a nivel global. Desde este punto de vista, las unidades legales y politicas subglobales pueden considerarse como encarnaciones alternativas de tal estructura -esto es, como interpretaciones concretas diferentes de los mismos sistemas abstractos de derechos humanos-. Puesto que las interpretaciones particulares estarán informadas por las circunstancias históricas y por las autocomprensiones de las sociedades organizadas alrededor de cllas, este modelo no ignora la contingencia ni la particularidad en su búsqueda de una identidad abstracta. Por el contrario, exige una apropiación crítica de lo aportado por la historia, la tradición, la cultura, las costumbres, etc. Cada unidad política debe decidir por sí misma qué elementos de lo dado buscará continuar perpetuando y cómo y cuáles deseará desconectar o transformar y de qué manera. Y si ha de ser una unidad política constitucional, con un Estado de Derecho, tanto internamente como en su relación con otras unidades políticas, los principios normativos que limitan esa apropiación incluirán alguna versión del sistema de derechos humanos básicos. Este modelo es claramente compatible con un grado sustantivo de soberanía local (incluso con esubsidiariedad»); no dicta por adelantado el nivel adecuado - municipal, provincial, Estado, regional, internacional o lo que sea - para la toma de decisiones, sólo defiende el principio general de que la gente debe poder decir algo en las decisiones políticas que afecten de forma significativa sus vidas ${ }^{31}$.

5. Debe quedar claro que esta forma de lograr la justicia internacional a través de una panoplia de encarnaciones de un sistema abstracto de derechos, en las que cada encarnación está limitada por un derechos cosmopolita institucionalizado, no deja las cosas tal y como estaban respecto a las diferencias políticas y culturales. Reconoce, claro está, que los significados concretos de los derechos y principios abstractos han de ser mediados a través de formas particulares de Sittlichkeit, o de vida ética, con sus concepciones diferentes del bien. Pero exige que, al margen de lo que dificran, todas las culturas políticas reconozcan los «mismos» derechos y principios básicos. Esto implica obviamente un cambio en la conciencia política de la mayoría, y aun diría de todas las sociedades existentes. Pero tal cambio no necesita realizarse por imposición violenta. Podríamos, por ejemplo, entrever un proceso en el que las sociedades primero aceptan - por las razones que sea: convicción, autointerés, «poder blando», etc.- el Estado de Derecho internamente e internacionalmente como, al menos, un modus vivendi, y luego lo aceptarían - a través de la socialización política y de la integración a lo largo de generaciones - por convicción, como parte del trasfondo compartido de vida

3. Éste fue el tema de una conferencia organizada por Dilip Gaokar en la Northwestem University en la primavera de 1996, a la que debo algunas de las ideas plasmadas en este texto.

3 Este es el principio que guia el repaso, por David Held, de la democracia política en la obra citada, nota 28. 
pública -es decir, es un proceso en el que un modus vivendi deviene eventualmente un orden ético-político- ${ }^{32}$. Esto no significa que los *imaginarios sociales» de todos los pueblos, las formas en las que piensan sobre sí mismo como pueblos vayan a corverger más de lo que lo hacen aquellos subgrupos o subculturas que tenemos en las sociedades pluralistas contemporáneas. Una única estructura legal y politica es evidentemente compatible con una gran multiplicidad de creencias, valores, proyectos de vida e identidades individuales. Una de las grandes ventajas de los derechos y de los principios abstractos es precisamente que permiten una enorme profusión de formas concretas de vida. Esto es, por decirlo de alguna manera, para lo que están diseñadas. Al mismo tiempo, los derechos humanos están pensados también para constreñir las präcticas locales e incluso a prevalecer sobre ellas cuando entran en conflicto con los derechos básicos tal como han sido elaborados, interpretados, implementados y aplicados por las autoridades legítimas relevantes.

6. El recién aludido momento interpretativo en cada rendición de derechos abstractos es una fuente ineluctable de desacuerdos razonables en el derecho y en la política. Dada la irreducible diversidad de cosmovisiones y formas de vida y, por tanto, de perspectivas interpretativas, siempre habrá desacuerdos razonables acerca de lo que requiera la justicia, y por tanto siempre habrá necesidad de compromiso y conciliación así como de consenso. Lo más que uno puede esperar a este respecto es que las discusiones públicas en las que estas diferencias se solventan sean informadas, razonadas y abiertas a todos, y que los procedimientos a través de los cuales se resuelvan finalmente sean, y se perciba que sean, iguales para todos los implicados. Es ésta una posición más profundamente procedimentalista que la de la teoría de la legitimidad del consenso de Habermas ${ }^{\jmath 3}$. Si los desacuerdos ético-políticos no son siempre, en principio, susceptibles de una única respuesta correcla, sus resoluciones procedimentalmente correctas no siempre serán stustantivamente aceptables, ni siquiera idealmente, para todas las partes. A menudo serán "racionalmente aceptables» para algunos grupos ético-culturales sólo indirectamente, esto es, sólo como resultado de procedimientos imparciales. Y a veces, cuando los intereses más básicos de los grupos están en litigio, determinados resultados, al margen de lo procedimentalmente correctos que sean, serán simplemente inaceptables. La conciliación y el acomodamiento de diferencias son especialmente importantes en este tipo de situación.

\footnotetext{
"A este respecto es parecido al proceso que Rawls describe acerca de la circunstancial aceptación de la tolerancia religiosa en la Europa posterior a la Reforma (PL xxii-xxvi).

${ }^{33}$ Bajo mi punto de vista, este tipo de procedimentalisno no es fundamentalmente incompatible con el importante papel que Habermas asigna al discurso razonado, porque es sólo y a través del discurso público como pueden articularse e interrogarse las diferencias éticas $-\mathrm{y}$ pueden resultar ser discursivamente irresolubles, esto es, desacuerdos razonables-. Los efectos racionalizadores sobre la calidad del discurso püblico que resultan de los procesos de institucionalización orientados a producir acuerdos informados y razonados no son en modo alguno confiscados con el reconocimiento de que el consenso no es sietnpre lograble, incluso bajo condiciones suficientemente ideales y a largo plazo.
} 